FERMILAB-Conf-99/085

\title{
Effect of the Beam-Beam Interactions on the Dynamic Aperture of the LHC at Collision
}

\author{
N. Gelfand, C. Johnston, T. Sen and W. Wan
}

Fermi National Accelerator Laboratory

P.O. Box 500, Batavia, Illinois 60510

April 1999

Presented at the Particle Accelerator Conference (PAC99),

New York, New York, March 29-April 2, 1999 


\section{Disclaimer}

This report was prepared as an account of work sponsored by an agency of the United States Government. Neither the United States Government nor any agency thereof, nor any of their employees, makes any warranty, expressed or implied, or assumes any legal liability or responsibility for the accuracy, completeness, or usefulness of any information, apparatus, product, or process disclosed, or represents that its use would not infringe privately owned rights. Reference herein to any specific commercial product, process, or service by trade name, trademark, manufacturer, or otherwise, does not necessarily constitute or imply its endorsement, recommendation, or favoring by the United States Government or any agency thereof. The views and opinions of authors expressed herein do not necessarily state or reflect those of the United States Government or any agency thereof.

\section{Distribution}

Approved for public release; further dissemination unlimited.

\section{Copyright Notification}

This manuscript has been authored by Universities Research Association, Inc. under contract No. DE-AC02-76CHO3000 with the U.S. Department of Energy. The United States Government and the publisher, by accepting the article for publication, acknowledges that the United States Government retains a nonexclusive, paid-up, irrevocable, worldwide license to publish or reproduce the published form of this manuscript, or allow others to do so, for United States Government Purposes. 


\title{
Effect of the Beam-Beam Interactions on the Dynamic Aperture of the LHC at Collision *
}

\author{
N. Gelfand, C. Johnstone, T. Sen, ${ }^{\dagger}$ and W. Wan,-FNAL, Batavia, IL 60510
}

\begin{abstract}
The dynamic aperture of the LHC at collision energy is limited by the field errors in the IR quadrupoles being built at FNAL and KEK. The $300 \mu$ rad crossing angle, incorporated in the design to reduce the effect of the long-range beam beam interactions, enhances the effect of the multipoles on the dynamic aperture. We have investigated the possibility of a different crossing angle with a more accurate modelling of the long-range interactions. Tune scans have been done to determine if a better choice of the tunes exists.
\end{abstract}

\section{INTRODUCTION}

At collision energy, nonlinear fields in the interaction region (IR) quadrupoles are the most important in determining the dynamic aperture of the LHC. The field quality "seen" by the beam while traversing these quadrupoles depends on the crossing angle of the beams at the IP. A study of the dynamic aperture, taking into account only the nonlinearities of the IR quadrupoles [1], had assumed a crossing angle of $300 \mu \mathrm{rad}$. This was based on an earlier study [2] which had determined this to be the optimum value when both the IR quadupole fields and beam-beam interactions were included.

On each side of the IP, there are fifteen long-range interactions, six of which occur in the drift space before the first quadrupole while the remaining nine occur in the triplet quadrupoles or in the drift spaces between them. Over the drift region the beams are round and the dimensionless separation $D_{\text {sep }}$ between the beams (measured in units of the rms beam size) stays nearly constant and equal to the crossing angle measured in units of the beam divergence at the IP. Once the beams are focused by the triplets, $D_{\text {sep }}$ is no longer constant. Figure 1 shows, for example, that with a crossing angle of $300 \mu \mathrm{rad}$, the separation varies between $7.8 \sigma$ to $13.6 \sigma$. In addition within the triplets, the beams are no longer round and the aspect ratio varies between 0.6 to 1.9. The phase advance from the IP to the locations of the long-range kicks varies from $82^{\circ}$ to $89^{\circ}$ through the drift section while within the triplets, it remains nearly constant at $90^{\circ}$. In order to reduce tracking time, earlier studies of the impact of the beam-beam interactions on the dynamic aperture made several approximations in treating the longrange interactions, viz. i) the phase advances between the long range kicks are negligible, ii) the beams are round at all locations of the long range kicks, and iii) the dimensionless beam separation stays constant. As we have seen, these assumptions break down in different regions within the IRs.

\footnotetext{
* Work supported by the U.S. Department of Energy under contract No. DE-AC02-76HO3000

$\dagger$ email: tsen@fnal.gov
}

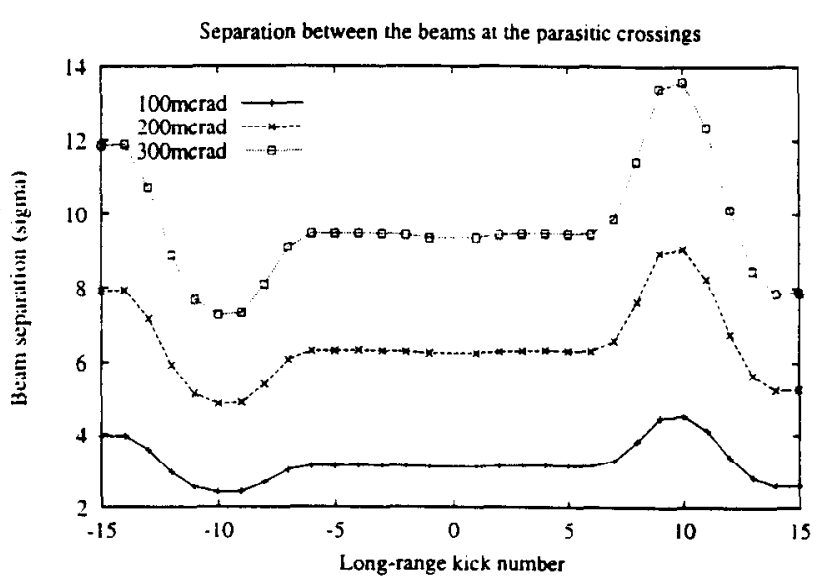

Figure 1: The separation between the two beams at each of the 30 parasitic crossings in a high luminosity IR for total crossing angles of 100,200 and $300 \mu \mathrm{rad}$. The separation, measured in units of the rms size of a beam, stays constant within the drift section (kicks from -6 to +6 ) but varies within the triplet quadrupoles.

In order to determine the optimum crossing angle more accurately, we have not made any of these approximations. Another reason for a second look at this issue is that the error harmonics in the IR quadrupoles have changed significantly since the last study was done. Specifically, reduced measurement errors have lowered estimates of high order harmonics by nearly an order of magnitude.

Among the several issues associated with the crossing angle geometry are: reduction in luminosity, orbit offset in IR quadrupoles which reduces the physical aperture, dispersion wave generated by the orbit offset, increase in the strength of the coupling, change in the beam-beam tune spreads, excitation of synchro-betatron resonances etc. The optimal crossing angle will ultimately be determined during operations. Our aim here is to study the impact of the choice of crossing angle on the required field quality of the IR quadrupoles and the complexity of correction schemes.

\section{LATTICE DESCRIPTION}

The version used is derived from the MAD lattice V5_l. The only lattice nonlinearities are the chromaticity correcting sextupoles and the systematic and random errors of the body harmonics of the triplet quadrupoles but not the uncertainties in the systematic nor the errors in the ends. Two different codes TEVLAT and MAD were used for tracking to $10^{3}$ turns.

Sixteen of the thirty two IR quadrupoles are to be built at 


\begin{tabular}{|c||c||c|}
\hline \multirow{3}{*}{$n$} & Normal & Skew \\
\cline { 2 - 3 } & {$\left[\left\langle b_{n}\right\rangle, d b_{n}, \sigma\left(b_{n}\right)\right]$} & {$\left[\left\langle a_{n}\right\rangle, d a_{n}, \sigma\left(a_{n}\right)\right]$} \\
& FNAL/KEK1 & FNAL/KEK1 \\
\hline 3 & $0, .3, .8 / 0, .51,1.0$ & $0, .3, .8 / 0, .51,1.0$ \\
4 & $0, .2, .8 / 0, .29, .57$ & $0, .2, .8 / 0, .29, .57$ \\
5 & $0, .2, .3 / 0, .19, .38$ & $0, .2, .3 / 0, .19, .38$ \\
6 & $0, .6, .6 / 0, .5, .19$ & $0, .05, .1 / 0, .10, .19$ \\
7 & $0, .06, .06 / 0, .05, .06$ & $0, .04, .06 / 0, .05, .06$ \\
8 & $0, .05, .05 / 0, .02, .03$ & $0, .03, .04 / 0, .02, .03$ \\
9 & $0, .03, .03 / 0, .01, .01$ & $0, .02, .02 / 0, .01, .01$ \\
10 & $0, .03, .03 /-1.0, .03, .01$ & $0, .02, .03 / 0, .01, .01$ \\
\hline
\end{tabular}

Table 1: Design field harmonics, at a reference radius of $17 \mathrm{~mm}$, of the IR quadrupoles to be built at FNAL and KEK. KEK1 refers to the first version. In the revised version (KEK2), $\left|\left\langle b_{10}\right\rangle\right| \leq 0.25$. Harmonics are expressed in units of $10^{-4}$.

Fermilab [3] and the other half will be built at KEK [4]. The designs of the cross-sections of the quadrupoles at the two laboratories differ and so do the expected error harmonics. Earlier plans had called for the magnets in one of the high luminosity insertions IR5 to be built at Fermilab while the magnets in the other high luminosity insertion IR 1 would be from KEK. In part due to the non-zero design value of $\left\langle b_{10}\right\rangle$ in the KEK magnets, it is presently proposed that the outer magnets $\mathrm{Q} 1$ and $\mathrm{Q} 3$ of each triplet be KEK magnets and the inner two magnets Q2a and Q2b (where the beam size is large) be Fermilab magnets.

\section{DYNAMIC APERTURE}

It is desirable to keep the minimum beam separation greater than $5 \sigma$ so the crossing angle must be greater than $100 \mu \mathrm{rad}$. We have calculated the dynamic aperture for various scenarios with crossing angles $\phi$ in the range $100 \mu \mathrm{rad} \leq \phi \leq$ $300 \mu \mathrm{rad}$.

In the initial studies, particles were tracked assuming that the error harmonics of all the triplet quadrupoles were those of the Fermilab quadrupoles. Subsequently the first, and later second, version of the KEK error harmonics was incorporated in the lattice. Table 2 shows the dynamic aperture calculated for all these different cases at a constant crossing angle of $300 \mu \mathrm{rad}$ but without the beam-beam interaction. The large value of $\left\langle b_{10}\right\rangle=-1$ in KEK1 leads to a significant drop in the dynamic aperture of about $2 \sigma$. The reduced value $\left(b_{10}\right)=-0.25$ in KEK2 improves the dynamic aperture by about $2 \sigma$. Mixing the magnets as described earlier further increases the dynamic aperture by $.6 \sigma$. In the following, all tracking calculations assume the mixed magnets scenario.

The beam-beam interactions are modelled in a similar fashion in TEVLAT and MAD, e.g. each kick is treated individually with the proper beam separation and the BassettiErskine expressions are used for kicks from non-round beams. As a check, the tune footprint with only the beam-

\begin{tabular}{|l|c|}
\hline IR Magnet Description & $\langle D A\rangle \pm \sigma_{\langle D A\rangle}$ \\
\hline FNAL only & $11.2 \pm 1.7$ \\
FNAL + KEK1 $\left(\left\langle b_{10}\right\rangle=-1\right)$ & $9.0 \pm .9$ \\
FNAL + KEK2 $\left(\left\langle b_{10}\right\rangle=-0.25\right)$ & $11.1 \pm 1.1$ \\
(no mixing ) & \\
$\begin{array}{l}\text { FNAL }+ \text { KEK2( }\left(\left\langle b_{10}\right\rangle=-0.25\right) \\
\text { (with mixing ) }\end{array}$ & $11.7 \pm 1.2$ \\
\hline
\end{tabular}

Table 2: Dynamic aperture (with TEVLAT) with only systematic and random errors in the body of the IR quadrupoles and without the beam-beam interaction.

beam interactions at a crossing angle of $300 \mu \mathrm{rad}$ was calculated with TEVLAT at amplitudes up to $6 \sigma$ and compared with that found by MAD [6]. The tune shifts with amplitude agreed to within $10 \%$.

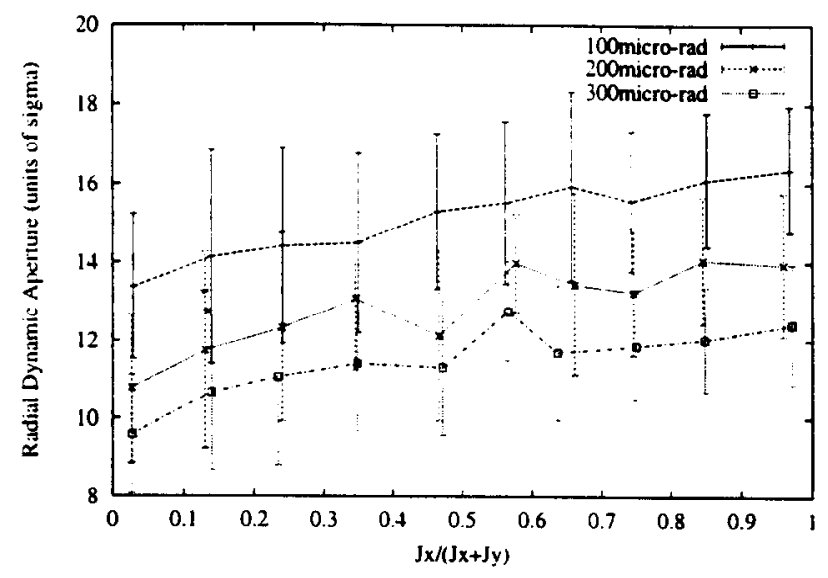

Figure 2: The dynamic aperture (in units of the rms beam size) calculated with TEVLAT at different aspect ratios in emittance space for different crossing angles. Triplet errors and the beam-beam interactions are included. The dynamic aperture clearly decreases with increasing crossing angle.

Figure 2 shows the dynamic aperture, averaged over 20 seeds, as a function of the transverse emittance ratio for three crossing angles. We observe that as the crossing angle increases from 100 to $300 \mu \mathrm{rad}$, the dynamic aperture decreases. We have also calculated the dynamic aperture at crossing angles of 150,225 and $250 \mu$ radians. Taken together, our results show that the dynamic aperture, even after including the beam-beam interactions, decreases nearly monotonically at all emittance ratios as the crossing angle increases.

Two different distributions of 20 random seeds were used with TEVLAT and MAD. Table 3 shows that the results from the two codes, both without and with the beam-beam interaction, are within 1-1.5 $\sigma$ of each other. Some of these differences may be due to the different seeds used and are within the statistical uncertainties of the averages. Both of these codes show clearly that the dynamic aperture decreases with increase in crossing angle. This table also 


\begin{tabular}{|l|c|c|}
\hline $\begin{array}{l}\phi \\
(\mu \mathrm{rad})\end{array}$ & $\begin{array}{c}\langle D A\rangle \pm \sigma_{\langle D A\rangle} \\
\text { TEVLAT } \\
\text { Beam-beam } \\
\text { OFF/ON }\end{array}$ & $\begin{array}{c}\langle D A\rangle \pm \sigma_{\langle D A\rangle} \\
\text { MAD } \\
\text { Beam-beam } \\
\text { OFF/ON }\end{array}$ \\
\hline 100.0 & $15.0 \pm 0.9 / 14.7 \pm 1.1$ & $13.8 \pm 1.1 / 13.3 \pm 1.0$ \\
200.0 & $13.8 \pm 0.8 / 12.4 \pm 1.1$ & $12.9 \pm 1.2 / 12.7 \pm 1.1$ \\
300.0 & $11.7 \pm 1.2 / 11.0 \pm 1.1$ & $11.6 \pm 1.6 / 12.0 \pm 1.3$ \\
\hline
\end{tabular}

Table 3: Dynamic aperture without and with the beambeam interaction calculated with TEVLAT and MAD at different crossing angles. The averages are over emittance space and over 20 random seeds.
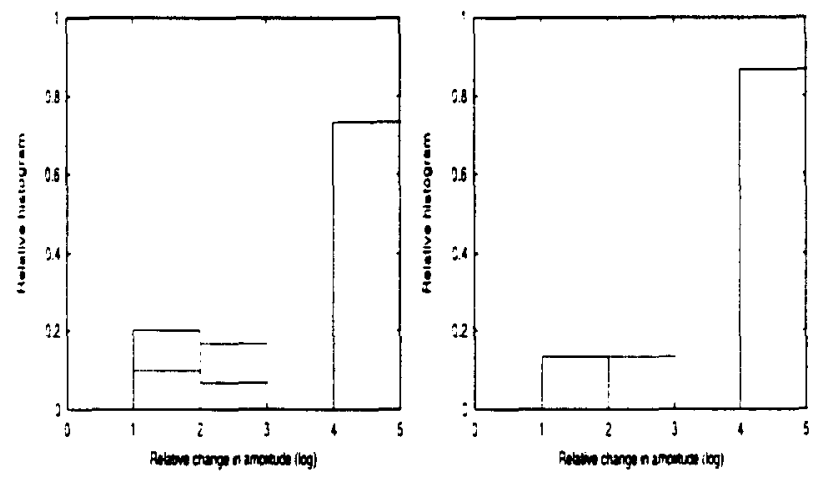

Figure 3: Normalized histograms of the relative amplitude growth (shown on a log scale) due to the resonances $2 \nu_{x}+$ $\nu_{y}=186$ (left) and $2 \nu_{x}+2 \nu_{y}=245$ (right). The histograms represent data from tracking with 30 seeds.

shows that the effect of the beam-beam interactions is always small compared to that of the IR quadrupole fields.

At a crossing angle of $300 \mu \mathrm{rad}$, tracking results have shown that higher order multipoles beyond $b_{10}, a_{10}$ also have an impact on the dynamic aperture [5]. We expect that the higher orders will have a smaller effect at smaller crossing angles due to the reduced feed-down.

\section{RESONANCES AND TUNE SCANS}

Among the low order resonances we have identified two, the skew third order resonance $2 \nu_{x}+\nu_{y}$ and the normal fourth order resonance $2 \nu_{x}+2 \nu_{y}$, as being driven strongly by the IR quadrupole nonlinearities. Figure 3 shows the normalized histograms, obtained with 30 seeds, of relative amplitude growth due to these resonances. For example, in more than $85 \%$ of the cases, the $2 \nu_{x}+2 \nu_{y}$ resonance leads to a larger than $10^{4}$ fold amplitude growth. Compensating these resonances with the MCBX and MCQS correctors may improve the dynamic aperture. Since the beam-beam interactions do not have a significant impact on the dynamic aperture at the crossing angles of interest, any correction scheme devised to correct for the nonlinearities of the IR quadrupoles should be adequate even when the beams col-

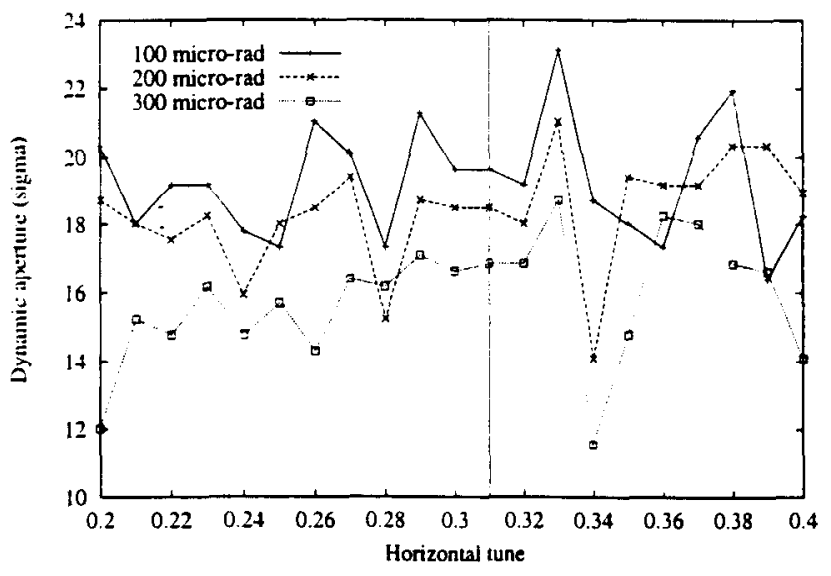

Figure 4: Tune scan of the dynamic aperture with a constant tune split, $\nu_{y}-\nu_{x}=0.01$. The vertical line represents the design tune, $\nu_{x}=0.31, \nu_{y}=0.32$.

lide.

A tune scan along the diagonal in emittance space and averaged over 10 seeds is shown in Figure 4 . The tune split is kept constant at $\nu_{y}-\nu_{x}=0.01$. This figure suggests there exist possible.tunes with dynamic apertures larger than at the chosen tunes. More detailed tracking studies, including tracking for off-momentum particles undergoing synchrotron oscillations, will be required to confirm the preliminary results shown here.

\section{CONCLUSIONS}

Our results suggest that the target dynamic aperture of $12 \sigma$ can be achieved with a $200 \mu \mathrm{rad}$ crossing angle, use of nonlinear correctors and the present design harmonics of the IR quadrupoles. It is currently expected that tuning shims will not be included in the final cross section design given the continuing improvement in field quality seen in the first model magnets [7]. At 200 $\mu \mathrm{rad}$, the shims are even less of a requirement and also the nonlinear correctors strengths are reduced. Our tune scans suggest that the dynamic aperture may be improved by a different choice of working point.

Thanks to J. Gareyte, H. Grote, J. Kerby, J.P. Koutchouk, M. Lamm, S. Peggs, R. Ostojic, F. Ruggiero, J. Strait, J. Wei and A. Zlobin.

\section{REFERENCES}

[1] J. Wei, et.al. Proc. EPAC 1998, p 380

[2] W. Chou, D. Ritson, Proc. PAC 1997

[3] J. Kerby et.al. Proc. ASC 1998, R. Bossert et.al., this conference

[4] Y. Ajima et.al.. Proc. EPAC 1998, p 2047

[5] N. Gelfand, this conference

[6] H. Grote, O. Meincke, LHC Project Note 161 (1998)

[7] J. Kerby, personal communication. 